\title{
reCHERches
}

Culture et histoire dans l'espace roman

25 | 2020

Mentir au théâtre

\section{Mensonges du pouvoir, mensonges de la scène dans le théâtre de Dario Fo}

\section{Cristina Vignali-De-Poli}

\section{(2) OpenEdition}

1 Journals

\section{Édition électronique}

URL : https://journals.openedition.org/cher/363

DOI : $10.4000 /$ cher.363

ISSN : 2803-5992

\section{Éditeur}

Presses universitaires de Strasbourg

\section{Édition imprimée}

Date de publication : 19 novembre 2020

Pagination : 113-123

ISBN : 979-10-344-0071-3

ISSN : 1968-035X

\section{Référence électronique}

Cristina Vignali-De-Poli, « Mensonges du pouvoir, mensonges de la scène dans le théâtre de Dario Fo », reCHERches [En ligne], 25 | 2020, mis en ligne le 01 octobre 2021, consulté le 17 novembre 2021. URL: http://journals.openedition.org/cher/363 ; DOI : https://doi.org/10.4000/cher.363 


\title{
Mensonges du pouvoir, mensonges de la scène dans le théâtre de Dario Fo
}

\author{
Cristina Vignali-De-Poli
}

Si parler d'un mélange de vrai et de faux, de réalité et d'invention au théâtre Orelève de la tautologie, dans le cas spécifique de l'œuvre de Dario Fo le mensonge mérite une réflexion à part. Le dramaturge, qui a été accusé à plusieurs moments de sa carrière d'avoir menti sur les sources de son théâtre, ainsi que sur certaines vérités ou figures historiques (il suffit de penser à son interprétation du giullare), se sert des mensonges du pouvoir - tels qu'il les perçoit - pour en faire des mensonges de la scène. Fo élabore une véritable poétique du mensonge et fait de ses propos théoriques une pratique théâtrale. Étant donné que le mensonge occupe une place non négligeable dans les écrits du dramaturge sur son théâtre, il nous paraît pertinent d'approcher son œuvre par ce biais. Les figures de menteurs peuplent également les pages de ses pièces. Nous tenterons de montrer comment le mensonge s'y révèle un instrument de dénonciation socio-politique. Enfin, nous étudierons comment le mensonge devient de manière spectaculaire le moteur narratif de Guerra di popolo in Cile (1973), une pièce entièrement fondée sur un mensonge au public.

«Il paradosso tragico dell'artista è di riuscire a far diventar credibile l'assurdo»: c'est ainsi que Fo communique, dans une interview à Giuseppina Manin (Fo 2007: 148), sa vision de l'artiste, une vision partagée par Franca Rame (femme du Prix Nobel, co-auteur, actrice et collaboratrice de son théâtre). L' «absurde» devient «crédible» par une déformation de la "réalité", par un mensonge. Le grotesque, que Dario Fo définit comme l' «atteggiamento abnorme, che smonta i meccanismi di una realtà fasulla» (Fo 1992: 35), constitue l'un de ses moyens privilégiés de déformation et de dénonciation de la réalité - politique ou sociale. Interrogé sur la différence qui sépare l'artiste et le faussaire, le dramaturge répondait ainsi:

Se si guarda la bravura tecnica la risposta può essere ardua. Ma invece è netta se si parla di creatività. Quello che rende un pittore o uno scultore un artista è il suo

1 Cristina Vignali-De-Poli, LLSETI, Université Savoie Mont Blanc 
saper cogliere la realtà in modo nuovo e diverso dagli altri [...]. Di tutto questo il falsario si limita a ricopiare la forma, anche se talora non senza talento. Però, è vero anche che fa parte del mestiere del pittore il mettere insieme il vero e il falso. (Fo 2007: 148)

Cette capacité du peintre à «mettre ensemble le vrai et le faux» caractérise, selon Giuseppina Manin, la conception même du théâtre de Fo - lequel, rappelons-le, fut non seulement dramaturge, acteur, écrivain, metteur en scène, décorateur de théâtre, scénariste, acteur cinématographique (à ses débuts), mais aussi peintre. Elle affirme en effet à propos de Fo: «Ogni sua commedia, ogni suo spettacolo è nato con questa stessa formula: mescolare, e non necessariamente in parti uguali, storia e fantasia, realtà e invenzione. Una storia dentro l'altra, e chi se ne importa se sono vere o no» (Fo 2007: 148-149).

La manipulation déformante du réel réalisée par Fo est liée non seulement au grotesque, mais s'étend aussi à l'histoire et aux sources qu'il emploie comme matière de son théâtre. Le dramaturge lui-même a souligné ce recours au "mensonge" dans son Manuale minimo dell'attore - ce "manuel" que Fo réalise après une expérience de leçons de théâtre et où il théorise de façon joyeuse sa pratique théâtrale: "Le nostre fonti non sono sempre attendibili, ma di certo sono quasi sempre affascinanti» (Fo 2009 : 367). Fo répète ici ce qu'il affirmait déjà dans le Prologue du Manuale où il évoquait en même temps les doutes soulevés par bien des critiques au sujet de la véracité de ses sources historiques:

Ebbene sì, è vero, ... spesso invento... ma attenzione, sia chiaro...le storie che mi fabbrico di sana pianta vi sembreranno ogni volta terribilmente autentiche... quasi ovvie... invece, quelle impossibili, paradossali, che giurereste inventate, sono al contrario tutte autentiche e documentabili. Sono un bugiardo professionista. E sono riuscito a far cascare in questa trappola del: «Non è vero, dubito» decine di prevenuti cacadubbi chiosatori». (Fo 2009: 4)

Mais pourquoi Dario Fo accorde-t-il autant de place au mensonge, tant au niveau théorique que, comme nous le verrons, au niveau de sa praxis théâtrale? La réponse est à trouver, nous semble-t-il, dans la nature politique de son théâtre: manipuler, voire déformer la réalité constitue la réponse théâtrale à cet autre genre de manipulation de la réalité que le pouvoir peut pratiquer. Face aux instruments dont le pouvoir se sert pour répandre ses mensonges - les médias, notamment - le théâtre de Fo se donne pour instrument le grotesque, ressort principal du mensonge, déformation du réel. Son théâtre se veut, en d'autres termes, un théâtre de déconstruction de la "vérité" du pouvoir, comme il l'affirme dans un chapitre de son Manuale minimo dell'attore, significativement intitulé Il falso, in teatro, è più attendibile:

[...] la lettura staccata e approfondita della conoscenza di tutto ciò che sta dietro ai fatti, ci permette oggi di reinventare in grottesco, in ironia o in tragico, tutto quello che la comunicazione diretta non riuscirà mai a darci. E il nostro dovere, o, se preferite, il nostro compito professionale, di autori, registi, gente di teatro, è riuscire a parlare della realtà violando lo schema standard col reagente della fantasia, con l'ironia, col cinismo della ragione. Così andiamo contro il 
programma e la strategia che il potere cerca di portare avanti: insegnare al pubblico a non usare mai il proprio senso critico [...]. (Fo 2009: 172)

$\mathrm{La}$ «stratégie» du pouvoir étant d'empêcher le peuple de raisonner, le grotesque permet de «réinventer» la réalité, d'en «violer les schémas standards», pour reprendre les mots du dramaturge: en d'autres termes, il faut dire le faux afin de parvenir à la «vérité» car l'art, et donc le théâtre, représente pour Fo « un mezzo e mai un fine. Un meraviglioso trampolino per arrivare ad altro: alla scienza, alla conoscenza, alla verità » (Fo 2007: 45). Par ce procédé paradoxal qui consiste à parvenir à la "vérité» par le biais du mensonge, le dramaturge entend faire un théâtre de la contre-histoire, de la contre-information et de la contre-culture (voir Soriani 2007), capable d'impliquer le spectateur, un spectateur qui doit être conscient de son propre rôle et non pas, pour reprendre une image qui est chère à Fo, vautré dans son fauteuil, "proteso solo a realizzare una tranquilla digestione» (Fo 2009: 147). Mentir au théâtre sert donc avant tout à réveiller les consciences.

Si l'intérêt de Fo pour le mensonge «aiguillon des consciences» ne fait aucun doute, la question de sa mise en œuvre mérite une attention toute particulière: en effet, par quels moyens la thématique du mensonge se déploie-t-elle dans son théâtre? Quelles sont les cibles visées? Et enfin, quelles fonctions précises a le mensonge dans l'esthétique et la pratique théâtrale du dramaturge?

Dans l'œuvre dramaturgique de Fo, rares sont les cas où le mensonge ne sert qu'à nourrir des jeux de théâtre, où le divertissement du public semble être le premier but de l'artiste. Ces exemples sont à repérer principalement dans ses pièces des années 1950 ou du début des années 1960, comme Non tutti i ladri vengono per nuocere (1958), où les mensonges des personnages adultères ne constituent qu'en arrière-fond la critique d'une Italie bigote et conservatrice. On évoquera également, pour ce type de mensonge à visée spectaculaire, les jeux vertigineux sur l'identité du personnage de Lungo (Gli arcangeli non giocano a flipper, 1959), qui ment à ses camarades en se montrant niais mais qui se révèlera ensuite - au public et au personnage féminin de La Bionda - tout autre que sot; on évoquera de même certains jeux d'identité dans Settimo: ruba un po' meno (1964), où le personnage de Nascimbene et de sa femme se mentent l'un l'autre, simulant la mort (Fo 2000: 119). Le mensonge dont ces personnages se servent permet éventuellement une mise en abîme, suggérant au public que ce qu'il voit sur scène ne correspond pas nécessairement à la vérité et qu'il lui faut donc - au théâtre comme dans la vie - se méfier des apparences, comme aime à le dire d'ailleurs haut et fort Enea, la femme protagoniste de cette dernière pièce (Fo 2000: 157).

Par le biais du miroir déformant du grotesque, ce sont souvent les mensonges du puissant que vise Dario Fo. Qu'il s'agisse d'un représentant du pouvoir politique, économique ou religieux, le puissant se voit obligé, le plus souvent malgré lui, à étaler sur scène ses mensonges, lesquels font ainsi l'objet de la satire du dramaturge. Les artifices pour mettre en scène les mensonges du puissant 
sont souvent liés à une situation de crise identitaire qui pousse celui-ci à se dévoiler. C'est ainsi que le Berlusconi bicéphale, ayant hérité d'une partie du cerveau de Poutine dans L'anomalo bicefalo (2003), avoue dans sa démence ses mensonges de politicien en même temps qu'il met à nu les outils de son pouvoir, les médias entre autres, instruments de contrefaçon du réel. De même, le Pape de la pièce Il Papa e la Strega (1989), en proie à une crise d'identité, dévoile l'hypocrisie de l'Église catholique en matière de politique des naissances ainsi que son pouvoir politique occulte en Italie. La crise d'identité de l'industriel Agnelli dans Claxon trombette e pernacchi (1981), suite à l'opération qu'il doit subir après un attentat et qui lui fait perdre son visage, est l'occasion de mettre en lumière le pouvoir dissimulé du dirigeant de Fiat et son influence dans le panorama politique italien de l'époque. Les lettres qu'Agnelli - faisant croire qu'il a été enlevé - envoie à la fin de la pièce aux représentants de la Démocratie Chrétienne, lettres calquées sur celles qu'envoya Aldo Moro lors de sa captivité, étalent au grand jour les mensonges qu'il utilisait pour gérer un pouvoir tout autant politique qu'économique. Enfin, la maladie de l'Amérique de La signora è da buttare (1967), en crise et bonne «à jeter», est jouée sur la scène d'un cirque, lieu par antonomase de l'imaginaire. L’Amérique se révèle le lieu des libertés mensongères pour Fo: on y est libre d'y émigrer mais sans être dignement accueillis; on y est libre de penser mais discriminé pour des raisons raciales, politiques, sexuelles sur lesquelles légifère une justice partiale; on y est trompé par le soi-disant bonheur consumériste.

Le mensonge préside également aux rapports entre homme et femme au sein du couple, où le sexe masculin s'impose, entre autres, grâce aux mensonges d'un langage qui diabolise l'autre sexe - il suffit de penser au Prologue de la pièce de 1977 Tutta casa, letto e chiesa (Fo 2000: 957-959), ainsi qu'à la vision terrifiante de l'orgasme donnée dans un des monologues de cette même pièce, Una donna sola (Fo 2000: 968). La disparité entre homme et femme relève également d'un mensonge dénoncé dans le monologue La mamma fricchettona (Tutta casa, letto e chiesa); la libération de la femme s'obtiendrait par le travail salarié, alors que dans la réalité elle doit faire un double travail: son activité professionnelle d'une part, ses tâches ménagères d'autre part (Fo 2000: 992). Mais Fo dénonce également les mensonges de la loi sur l'avortement que la femme subit en premier, comme c'est le cas pour la femme protagoniste d'un autre monologue de la pièce, Abbiamo tutte la stessa storia (Fo 2000: 1001). Victime de multiples mensonges qui la pénalisent face à l'homme, la femme de Fo - et Rame - se ment également à elle-même, du moins jusqu'à ce qu'elle apprenne à se rebeller. La femme protagoniste d'Una donna sola ne cesse de répéter initialement que son mari ne lui fait manquer de rien à la maison, se mentant à elle-même pour ne pas sombrer dans la dépression alors que son présent n'est fait que de violence et d'humiliations. Dans ce cas, pour utiliser les mots de Mario Lavagetto, "[1]a bugia non è che un gesto di difesa, lo schermo momentaneamente opposto a una realtà accecante» (Lavagetto 1992: 240). Ces mensonges à elle-même ainsi que 
les leurres que sont les biens de consommation ne peuvent en rien guérir cette femme seule et prisonnière.

Le mensonge est également celui d'une classe dominante qui l'utilise pour imposer sa culture: c'est ainsi que dans la pièce Hellequin, Harlekin, Arlekin, Arlecchino (1985) Fo met en scène un Arlequin présenté comme personnage historique et qui n'a rien en commun avec l'Arlequin serviteur de Goldoni qu'une longue tradition culturelle a imposé. Pareillement, dans la pièce consacrée à saint François, Lu Santo Jullàre Françesco (1999), Fo joue un François irrévérencieux qu'il dit avoir tiré non pas de l'histoire officielle édulcorée du saint, mais de vieilles fables populaires des campagnes de l'Ombrie (Fo 2000: 880-881). Dans Mistero Buffo (1969), le dramaturge dénonce également la culture dominante, accusée de falsifier la culture populaire au sein même de l'école, comme c'est le cas pour le célèbre poème Rosa fresca aulentissima dont les manuels scolaires passent sous silence les origines populaires et la signification paillarde (Fo $2000: 217$ ).

Le mensonge ne concerne toutefois pas seulement ceux qui détiennent un pouvoir, mais aussi ceux qui essaient de parvenir par la tromperie à une forme de pouvoir. L'exemple le plus probant est le Christophe Colomb de la pièce Isabella, tre caravelle e un cacciaballe (1963), un Colomb dont le trait principal est d'être, comme l'indique le titre, «cacciaballe», c'est-à-dire menteur. Colomb, qui ment aux puissants souverains d'Espagne pour financer ses voyages, ainsi qu'à ses compagnons de voyage par opportunisme, sera cependant puni; Fo entend ainsi condamner le mensonge des arrivistes et des opportunistes qui mentent pour leur strict intérêt personnel.

À côté de ces menteurs qui se servent de leur pouvoir (ou essaient de le faire) se trouve une autre catégorie d'individus qui mentent pour dénoncer le pouvoir. Ils se déguisent et, par cette forme de mensonge qu'est la dissimulation de leur vraie identité, deviennent les pourfendeurs de l'injustice, de la corruption, de l'inégalité des chances, voire du crime d'État et de ses mensonges. L'exemple le plus éloquent est celui du Fou, protagoniste de Morte accidentale di un anarchico (1970). Son identité toujours changeante, et mensongère donc - car il se déguise et joue le rôle tour à tour d'un commissaire au téléphone, d'un juge, d'un capitaine de la police scientifique, d'un évêque - lui permet de dénoncer les mensonges divers d'un État responsable de la mort de l'anarchiste Pinelli à la préfecture de Milan suite à l'attentat de Piazza Fontana. De même, dans la pièce Non si paga! Non si paga! (1974), la fausse grossesse de Margherita, qui en réalité cache sous son vêtement la «dépense prolétaire» de son amie Antonia - à savoir les courses qu'elle n'a pas payées en signe de désobéissance civile et de protestation contre le renchérissement de la vie - vise à dénoncer une condition ouvrière désastreuse due à la conjoncture économique pénalisante du début des années 1970. Le travestissement en bonne sœur d'Enea, la femme croquemort de Settimo: ruba un po' meno, donne lieu à une scène où est dénoncée entre autres l'avidité de l'Église catholique (Fo 2000: 168). Ce mensonge qu'est la dissimulation de l'identité d'un personnage permet au dramaturge de faire émerger des vérités sur la société telle qu'il la perçoit. 
Une place à part dans notre étude doit être faite à Guerra di popolo in Cile. Cette pièce militante, dont les recettes furent versées à la résistance chilienne, fut jouée la première fois au Palais des Sports de Bolzano le 20 octobre 1973, après trente jours de répétitions du collectif théâtral $\mathrm{La}$ Comune (dont Fo et Rame faisaient alors partie). L'intérêt que nous portons à Guerra di popolo in Cile est dû au fait que toute la pièce, née en réaction au putsch du général Pinochet du 11 septembre 1973, se fondait sur un mensonge.

Après une ouverture portant sur le récit du putsch chilien accompagné de l'enregistrement des dernières heures d'émission de la radio du Movimiento de Izquierda Revolucionaria (MIR) - avec en arrière-fond les vrombissements d'avions et les explosifs - la pièce prenait la forme d'un spectacle de chansons et de discours sur la résistance au Chili. Puis, progressivement, un nouveau spectacle se superposait au spectacle sur la résistance chilienne: des individus - des policiers apparemment - finissaient par faire irruption dans la salle pour y arrêter des activistes de la gauche extra-parlementaire; Fo faisait croire au public qu'un coup d'État avait lieu en Italie et le public tombait dans le piège. Le mensonge n'était mis à nu qu'au dénouement lorsque l'Internationale était entonnée non seulement par les acteurs "sur scène", qui invitaient le public à se joindre au chant et à bien veiller au sens des mots chantés, mais aussi par les soi-disant policiers qui avaient fait irruption. En chantant avec les acteurs, les policiers dévoilaient leur vraie nature d'acteurs et, de cette manière, révélaient la fiction scénique. Dévoilement de la vérité (la vérité de la fiction théâtrale) et chant révolutionnaire coïncidaient alors - en réalité la chanson a bien souvent une fonction de protestation et de vérité chez Fo. La mise en scène du mensonge coûta d'ailleurs à notre dramaturge une arrestation en bonne et due forme lors de la représentation de la pièce à Sassari, le 10 novembre 1973, parce qu'il s'était opposé à ce que de vrais policiers entrent dans la salle pour faire cesser la représentation. La fiction du mensonge devenait d'une certaine manière réalité.

Le mensonge du coup d'État n'avait été pensé que dans une troisième étape de la conception de la pièce (Nepoti et Cappa 1997: 105), au moment où Fo se rendait compte de la nécessité d'introduire un élément susceptible de secouer ses spectateurs. Le dramaturge entendait exploiter la charge émotionnelle du mensonge, sa force de "provocation» (Nepoti et Cappa 1997: 105). Il écrira d'ailleurs à propos du théâtre grec des origines: «E quanto più bravo [il drammaturgo] si dimostrava nel provocarlo [il pubblico] e nel metterlo in crisi, maggiore era la stima e l'applauso che gli si tributava [...]» (Fo 2009: 175). Provoquer le public pour en mettre en crise les certitudes et le pousser à l'action, «nega[re] la passività del pubblico» (Fo 1973: 7) : tel était le premier objectif affiché dans la Nota editoriale de l'édition Bertani de Guerra di popolo in Cile. Fo obligeait le public à voir en Italie ces mêmes mécanismes de l'impérialisme et du capitalisme qui s'étaient exprimés avec le putsch et la violente contre-révolution chilienne (Fo 1973: 7). Contre la tendance des médias à passer les problèmes sous silence, la pièce entendait les "dire", comme le précisait le personnageDario dans le monologue initial: 
a noi del Cile vogliamo parlarvene e come! [...] [N]oi pensiamo che sia [...] importante riuscire a scoprire e capire insieme quale enorme insegnamento possa essere per tutti noi l'esperienza cilena! Quale monito, quale esempio. (Fo 1973: 26)

Mentir pour faire valoir l'expérience chilienne comme enseignement et, en luttant contre le silence imposé par les médias, dire au public une vérité. «Recitare il falso per insegnare il vero» (Rame, Fo 2009: 238) est d'ailleurs significativement le titre du chapitre que Franca Rame consacre à Guerra di popolo in Cile dans son autobiographie Una vita all'improvvisa.

Dans le contexte hautement politisé de l'Italie du début des années 1970, il est légitime de se demander, à ce stade, quel type de vérité le spectacle entendait véhiculer. Une réponse éloquente à cet égard vient de la Nota editoriale citée précédemment; la note précise que la pièce visait à mettre en lumière «la verità storica» selon laquelle "le classi dominanti non abbandonano pacificamente il potere» car «il capitale non appena si sente minacciato ricorre alla strage» (Fo 1973: 7). On retrouve le souci constant chez Fo de souligner une division nette des classes, entre oppresseurs et oppressés, entre détenteurs du capital et du pouvoir d'une part, et, exploités d'autre part. En d'autres termes, cette "vérité", loin d'être apolitique, était l'expression de l'adhésion au concept marxiste de la lutte des classes - à une époque où Fo s'était définitivement éloigné du PCI pour se rapprocher des organisations de la gauche extraparlementaire.

Mentir signifiait provoquer pour faire émerger une vérité autre par rapport à celle des classes dominantes et du parti majoritaire de la Démocratie Chrétienne, et secouer avec cette "vérité" un public qui ne pouvait demeurer passif dans l'Italie des années de plomb. Il s'agissait d'une invitation dont le but était d'ailleurs expliqué au cours des débats animés avec le public qui suivaient les représentations de Guerra di popolo in Cile: «far sì che ognuno si eserciti a non esser preso all'improvviso dentro qualsiasi trappola del potere costituito » (Rame et Fo 2009: 249).

Il convient maintenant de voir comment la scène de Guerra di popolo in Cile dit le mensonge. Si l'on s'intéresse aux outils exploités scéniquement, on se rend compte tout d'abord que Fo y fait un usage bien plus massif que dans toute autre pièce de différents appareils permettant l'irruption du monde extérieur dans l'espace traditionnellement ouaté de la salle de spectacle - encore que la pièce naisse à une époque où le dramaturge a déjà déserté les salles de théâtre considérées comme bourgeoises pour choisir des espaces autres, plus près du peuple.

Outre l'emploi du haut-parleur et d'un système d'amplification du son, Fo y exploitait un appareil émetteur-récepteur, à la fois micro pour la salle et radio permettant de capter des sons provenant de l'extérieur; par ce moyen, il arrivait à créer artificiellement un lien entre le dehors et le dedans de la salle, où la réalité semblait se déverser. Après avoir entendu l'enregistrement de la radio du MIR renvoyant, au tout début de pièce, au contexte de la lutte armée et de la répression au Chili, le public entendait, par le biais de l'émetteur-récepteur 
que le personnage-Dario portait au cou et dont le son était amplifié, d'autres voix et bruits provenant de l'extérieur de la salle; Fo faisait entendre ainsi des forces de police situées apparemment tout près du lieu de la représentation et qui semblaient prêtes à intervenir. Ce lien entre dehors et dedans faisait de la salle un espace de lutte, créant un parallèle angoissant entre le putsch chilien et le putsch italien (inventé).

L'invention d'un putsch dans la Péninsule était d'autant plus crédible que les Italiens avaient bien en mémoire la tentative avortée de coup d'État de décembre 1970 qui avait vu des débuts d'actions paramilitaires dans plusieurs villes italiennes et qui, au bout de quelques heures, ne fut mystérieusement stoppé que par ordre de son principal organisateur, le prince Borghese. L'année 1973 est d'ailleurs celle de la sortie (environ six mois avant le putsch chilien) du film de Mario Monicelli Vogliamo i colonnelli, film qui s'inspire aussi bien de la dictature des colonels en Grèce (1967-1974) que du putsch avorté de Borghese.

Il faut rappeler en outre que le public de Fo était habitué aux actions policières visant à entraver son travail de dramaturge militant - ce sur quoi joue le dramaturge dans sa pièce: "Speriamo che si allontanino, certe volte lo fanno apposta di mettersi vicino... lo sanno che noi usiamo il Binson... fanno opera di disturbo...» (Fo $1973: 28$ ).

Les commentaires des acteurs donnent un sens et en même temps un espace scénique central aux interceptions provenant de l'émetteur-récepteur. Quand les messages sibyllins de la police qui arrivent par l'émetteur sont commentés, la pièce censée se dérouler s'interrompt, l'attention du public se focalise alors sur le contenu de ces messages énigmatiques et la (fausse) menace semble peser plus lourdement dans la salle. Ces commentaires des acteurs - qui participent pleinement du mensonge - semblent répondre à trois fonctions principales: ils contribuent à amplifier l'idée de menace, deviennent de véritables instruments de dénonciation du pouvoir et de ses émanations ${ }^{2}$, et, surtout, permettent au dramaturge de jouer sur la limite troublante entre vérité et mensonge. Lorsque, tout à coup, on n'entend plus aucune voix de policiers sortir de son appareil, le personnage-Dario affirme en effet: «Beh, approfittiamone per andare avanti. A questo punto, però, vi vogliamo dire la verità: quest'ultima intercettazione l'abbiamo organizzata apposta noi con la polizia per trovare un buon pretesto alla presentazione della prossima canzone...» (Fo 1973: 34-35).

Le spectateur ne sait plus si le personnage-Dario fait de l'ironie ou bien s'il dit la vérité; quoi qu'il en soit, cette affirmation est un mensonge dans le mensonge, bien que présenté par l'acteur comme un aveu de vérité. Ce commentaire

2 Il suffit de penser à la dénonciation de l'appareil militaire italien excessivement fourni mais inefficace (Fo 1973: 35) ou à la dénonciation des conflits internes entre Polizia et Carabinieri (Fo 1973: 34), forces concurrentes alors qu'elles devraient être solidaires de par leur mission - cette dernière dénonciation se retrouve d'ailleurs également dans Morte accidentale di un anarchico. 
paradoxal, qui donne (a posteriori) la clé du jeu scénique, invite le public à aiguiser son esprit critique pour savoir discerner le vrai du faux.

La mise en scène du mensonge a lieu également par d'autres artifices scéniques qui se multiplient au fur et à mesure que le spectacle avance, créant une situation chaotique qui peu à peu semble paralyser le spectacle sur la résistance chilienne. En tout premier lieu, on remarquera la multiplication des personnages - réels ou seulement évoqués - présentés comme extérieurs à la pièce voire à la salle, mais étant en réalité de vrais acteurs. Les spectateurs voient entrer dans la salle un homme qui se présente comme policier, un autre comme commissaire. Le public est abusé et croit véritablement que ces hommes sont des représentants de la loi. Qu'il s'agisse d'un policier en uniforme, d'un commissaire qui dit pouvoir entrer dans la salle grâce au mandat d'un juge, ou d'un (faux) spectateur dans le parterre (Fo 1973: 81-83), dans tous les cas il ne s'agit pas de simples figurants; ces personnages deviennent au contraire l'occasion d'une interaction avec les acteurs 'sur scène ' et avec le public. Des micro-situations d'échange (ou de pseudo-échange) entre les faux représentants de la loi, les acteurs, voire le public, naissent alors autour de questions qui étaient épineuses pour le Collectif de Fo à l'époque. Ainsi le personnage-Dario se dispute avec le faux policier au sujet de l'intrusion de la police dans la salle, lieu public d'après le policier, lieu privé d'après l'acteur (Fo 1973: 81); ou bien il s'adresse directement au public pour justifier sa sortie de scène en faisant allusion à un problème récurrent lors de ses représentations: "Scusatemi un attimo, sarà la solita grana dell'agibilità o del fatto dei minori di diciotto anni, scusatemi vengo subito.» (Fo 1973: 82).

La multiplication des personnages détermine une multiplication des interruptions du spectacle que les acteurs font semblant de vouloir jouer. Ces interruptions de plus en plus récurrentes font également partie de la construction du mensonge et tirent leur efficacité scénique du fait qu'elles sont mises en valeur par les personnages "sur scène", portant l'attention des spectateurs sur la menace extérieure. On rappellera le moment où le personnage-Franca interrompt le personnage-Dario en expliquant les raisons de cette coupure qui ont à voir avec l'organisation du putsch: "Scusa se ti interrompo, ma uno dei ragazzi del servizio d'ordine dice che sono passati un sacco di automezzi, una autocolonna della polizia e anche camion e mezzi cingolati dell'esercito» (Fo 1973: 82).

Parmi ces interruptions du jeu scénique on comptera également ces brèves communications de service entre acteurs et membres du service d'ordre du Collectif, communications qui deviennent de véritables situations théâtrales, parties intégrantes de la mise en scène du mensonge. C'est ainsi que le personnage-Dario s'adresse à ses camarades, formulant en même temps un commentaire qui souligne l'étrangeté de la situation : «Ehi, compagni del servizio d'ordine non avete fermato l'agente?! Ma dove sono andati» (Fo 1973: 81).

Le mensonge se construit aussi par le contraste entre les positions de Dario et celles de Franca, principaux acteurs de la pièce, mari et femme dans la vie privée. Tandis que le personnage de Dario cherche à minimiser ce qui se passe dans la 
salle $^{3}$, celui de Franca ne cesse de s'inquiéter et de manifester son inquiétude au public $^{4}$. Cette mise en scène de l'opposition des points de vue dans le couple, tout en donnant une couleur comique à la scène, permet de mettre encore une fois au centre de l'attention du public les modalités du (faux) coup d'État.

Le mensonge arrive à son acmé quand le commissaire appelle un par un les militants dans la salle - de vrais militants de la gauche extra-parlementaire qui joueront le jeu de répondre à l'appel et de monter sur scène en plein accord avec le Collectif. Le personnage-Dario noue un pacte avec son public juste avant l'entrée du commissaire:

Mi raccomando, come se niente fosse, nessuno che faccia lo spiritoso o che si metta a gridare, sfottere o fischiare. Ricordate che può essere un ottimo pretesto per farci togliere il permesso di agibilità. E non farci più recitare. (Fo 1973: 83-84)

Ce pacte permet d'une part d'établir une relation inédite entre acteurs et public, invité à participer positivement au bon déroulement de cette nouvelle situation; en brisant le quatrième mur, l'acteur invite d'autre part son auditoire à la responsabilité et à la défense d'une même cause (révolutionnaire) qui semble unir spectateurs et troupe théâtrale.

Le dramaturge réalise une opération somme toute unique au sein de sa production: les acteurs qui jouent "sur scène" s'identifient pendant presque toute la pièce à leurs personnages d'acteurs consternés par les signes du (faux) putsch. On pourrait penser ainsi que Guerra di popolo in Cile est une pièce éloignée du théâtre épique tel que Fo le conçoit: un théâtre où l'acteur, au lieu de s'identifier avec son personnage, le raconte et le présente (Fo 1992: 126). En réalité, avec la fin, le jeu se révèle extraordinairement épique: si l'acteur s'est identifié à son personnage, c'est non seulement pour faire adhérer le spectateur au mensonge du coup d'État, mais aussi et surtout pour faire retentir avec plus de force le moment conclusif de la pièce où ce mensonge est mis à nu - et où les apparences se sont montrées spectaculairement trompeuses.

Cette pièce de Fo est également unique par les réactions qu'elle a pu susciter dans le public. Chiara Valentini écrivait à ce propos:

[...] a Torino, un ragazzo si è mangiato dieci pagine della sua agenda, fitte di indirizzi che giudicava compromettenti. A Merano, uno studente ha tentato di buttarsi da una finestra, rompendo anche un vetro. A Nuoro, addirittura, dove erano arrivati due pullman di pastori da Orgosolo, c'è stato chi, alla vista del

3 «Beh, non c'è niente di strano, non facciamoci prendere dalla psicosi per carità, se no passa un gatto e diciamo che era un generale mimetizzato. Staranno facendo delle normali esercitazioni »; ou encore: «Calma compagni, niente di grave per quanto un po' strano» (Fo 1973: 82).

4 «Già, che strano, i telefoni bloccati, la radio muta, le autocolonne che transitano a tutta birra. Gli ordini della polizia di prima fra le autocivette di andare alla caserma, il poliziotto poco fa, addirittura un vicequestore che viene a scomodarsi per dare un'occhiata alla ricerca di due o tre ladruncoli»; ou encore: "Per me c'è sotto qualcosa che puzza. È chiaro che il fatto dei ladri era del tutto un pretesto. Chi sa chi cercano?» (Fo 1973: 82-83). 
finto commissario, ha fatto lampeggiare le lame dei coltelli con cui si taglia il formaggio. (Valentini 1973)

Le but de la pièce qui consistait à faire réagir le public pour qu'il ne cède pas à la tentation de se trouver vautré dans son fauteuil semblerait ainsi avoir été atteint grâce à une utilisation du mensonge sciemment maîtrisée.

Au terme de notre étude, force est de constater à quel point mensonge et vérité s'entremêlent dans la dramaturgie de Dario Fo et Franca Rame pour potentialiser un théâtre qui se veut engagé. La notion de "vérité", telle que le dramaturge la conçoit, se forme par opposition à l'information, à la culture et à l'histoire officielles. La vérité que les mensonges de Fo veulent faire émerger est donc une vérité alternative à celle des classes dominantes. C'est la vérité, non moins politisée, de tous ceux qui, pour Fo, n'ont pas eu les instruments (ou le pouvoir) pour la faire sortir de l'ombre.

\section{Bibliographie}

Fo D., 1973, Guerra di popolo in Cile, Vérone, Giorgio Bertani Editore.

Fo D., 1984, Non tutti i ladri vengono per nuocere, dans Le commedie di Dario Fo VI, Turin, Einaudi, 1984.

Fo D., 1992, Fabulazzo. Il teatro, la cultura, la politica, la società, i sentimenti: articoli, interviste, testi teatrali, fogli sparsi, 1960-1991, Milan, Kaos edizioni.

Fo D., 2000, Teatro, Turin, Einaudi.

Fo D., 2006a, Hellequin, Harlekin, Arlekin, Arlecchino, Milan, Fabbri Editori.

Fo D., 2006b, Il Papa e la Strega, Milan, Fabbri Editori.

Fo D., 2006c, Isabella, tre caravelle e un cacciaballe, Milan, Fabbri Editori.

Fo D., 2006d, L'anomalo bicefalo, Milan, Fabbri Editori.

Fo D., 2006e, La signora è da buttare, Milan, Fabbri Editori.

Fo D., 2007, Il mondo secondo Fo. Conversazione con Giuseppina Manin, Parme, Guanda Editore.

Fo D., 2009, Manuale minimo dell'attore, Turin, Einaudi.

Lavagetto M., 1992, La cicatrice di Montaigne. Sulla bugia in letteratura, Turin, Einaudi.

Nepoti R., Cappa M., 1997, Dario Fo, Rome, Gremese Editore.

Rame F., Fo D., 2009, Una vita all'improvvisa, Parme, Guanda Editore.

Soriani S., 2007, Dario Fo. Dalla commedia al monologo (1959-1969), Pise, Titivillus Edizioni.

Valentini C., 1973, "Pum pum! Il Questore” (Panorama, 22 novembre 1973), in Archivio Franca Rame Dario Fo, <http://www.archivio.francarame.it/scheda. asp?id=025804\&from $=1 \&$ descrizione $=$ CILE $>$ [consultée le 14 mars 2012]. 